\title{
PERIODICAL NONUNIFORM INDIVIDUALLY SAMPLED SWITCHED-CAPACITOR CIRCUITS
}

\author{
J. L. Ausín ${ }^{\prime}$, J. F. Duque-Carrillo ${ }^{1}$, G. Torelli ${ }^{2}$, E. Sánchez-Sinencio ${ }^{3}$, and F. Maloberti ${ }^{2}$ \\ ${ }^{1}$ Dept. of Electronics and Electr. Eng., University of Extremadura, (06071) Badajoz, Spain. \\ ${ }^{2}$ Dept. of Electronics, University of Pavia, Via Ferrata 1, I-27100 Pavia, Italy. \\ ${ }^{3}$ Dept. of Electrical Engineering, Texas A\&M University, College Station, TX 77843, USA.
}

\begin{abstract}
This paper presents a technique for programmable switchedcapacitor (SC) filters based on periodical nonuniform individual sampling (PNIS) of each SC structure in the filter. It is shown how very wide range of programmability in all circuit parameters can be obtained, whereas the dynamic range degradation is kept minimum. Experimental results obtained from a $1.2 \mu \mathrm{m}$ CMOS second-order SC filter are given.
\end{abstract}

\section{INTRODUCTION}

Switched-capacitor (SC) circuits have demonstrated an enormous potentiality for the monolithic implementation of many systems, though the most extended application of these circuits is filtering [1]. Among others, one of the most attractive features of SC circuits is the programmability. In general, programmable SC circuits incorporate digitally-programmable capacitor arrays (DPCAs), in such a way that the effective capacitance value is controlled by an applied digital code. However, programmable SC circuits based on DPCAs suffer from limited programming versatility, increase in parasitic capacitances and, even more importantly, dynamic range degradation. This dynamic range degradation, which is a common drawback in most programming techniques, arises as a consequence of the variable nature of the circuit and prevents optimum capacitor value scaling.

In this paper, a technique, referred to as periodical nonuniform individual sampling (PNIS), for obtaining very wide programmability ranges in all circuit parameters, is proposed. It is shown how no dynamic range degradation exists when some parameters are changed. The application of PNIS to timemultiplexed (TM) SC filters is also shown. Experimental results obtained from a $1.2 \mu \mathrm{m}$ CMOS test-chip prototype, that demonstrated the potentiality of the technique, are provided.

\section{SC CIRCUITS WITH PERIODICAL NONUNIFORM INDIVIDUAL SAMPLING}

Periodical nonuniform individual sampling is a new way to control SC networks. According to this technique, any individual $\mathrm{SC}$ structure is active during only $p$ clock periods over a given number $m$ of clock pulses $(p \leq m)$. The $p$ active periods can be differently distributed within the overall repetition period $m T_{s}\left(T_{s}\right.$ being the master clock period), however, in this paper we assume that these $p$ active periods are concentrated at the beginning of each sequence of $m$ clock pulses.

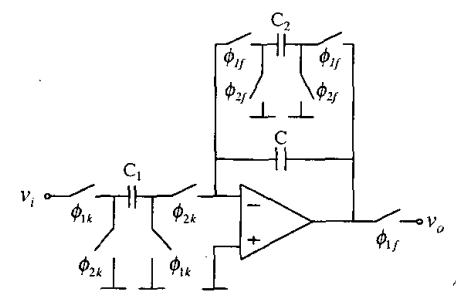

(a)

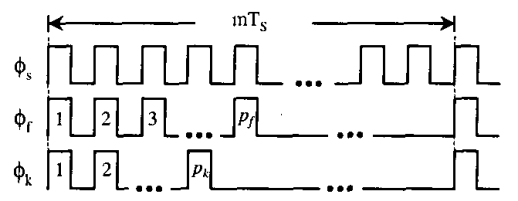

(b)

Fig. 1. Damped SC integrator (a) and clock signals for PNIS (b).

Figure 1 shows the PNIS technique applied to a simple damped $\mathrm{SC}$ integrator. In this figure, $\phi_{\mathrm{f}}$ and $\phi_{\mathrm{k}}$ represent the clocks of the switched-capacitors $C_{2}$ and $C_{l}$, respectively. Both $\phi_{\mathrm{f}}$ and $\phi_{\mathrm{k}}$ are implemented as two non-overlapping phases $\left(\phi_{1 \mathrm{f}}, \phi_{2 \mathrm{f}}\right.$ and $\phi_{1 \mathrm{k}}, \phi_{2 \mathrm{k}}$, respectively), and are derived from a master clock $\phi_{s}$. Notice that the switched-capacitors $C_{j}$ and $C_{2}$ are active during $p_{k}$ and $p_{f}$ pulses, respectively, in the overall repetition period $m T_{s}$. We can observe that for the simple circuit in Fig. 1, the PNIS technique is a sort of pulse width modulation applied to each equivalent SC resistor, which substantially results in the possibility of programming the resistor value by properly choosing the number of active pulses. As we will see shortly, when the technique is used in a more complex network including feedback loops, it becomes a powerful tool for a digitally programmable control of linear filters.

For high sampling frequency as compared with the maximum frequency of the input signal (i.e., for $m \omega T_{s} \ll 1$ ), it can be demonstrated that the DC gain $(k)$ and the pole frequency $\left(\omega_{o}\right)$ of the damped integrator in Fig. 1 are given, respectively, by

$$
\begin{gathered}
k=\frac{p_{k}}{p_{f}} \cdot \frac{C_{1}}{C_{2}} \\
e^{j \omega_{o} n i T_{s}}=\left(\frac{C}{C+C_{2}}\right)^{p_{f}}
\end{gathered}
$$


As expected, eqs. (1) indicate that the parameters of the damped SC integrator response in Fig. 1, can be programmed by controlling the effective sampling frequency of the individual SC branches (i.e., by means of the number of active clock pulses $p_{k}$ and $p_{f}$ ). This fact allows one to implement programmable SC circuits with no need for incorporating any capacitor array and, therefore, to avoid the above mentioned constraints associated with DPCA programmability. More programming versatility is also provided, by controlling the values of $p_{k}$ and $p_{f}$. The symbols $p_{k}$ and $p_{f}$ will be referred to as the gain and frequency programming indexes, respectively, and $p_{q}$ as the $Q$-factor programming index, when existing. In principle, every programming index can vary, at most, between 1 and the value of the general programming index $m$.

According to the clock signals in Fig. 1(b), in general, the transfer function of an SC circuit operating with a PNIS will consists of the sum of $L$ partial transfer functions, where $L$ accounts for the number of clock pulses in which the output signal is sampled. The partial transfer functions can be represented by the following expression:

$$
H_{j}(z)=\frac{V_{o}^{j}}{V_{i}}=\sum_{l=1}^{v} H_{j l}(z)
$$

where $H_{j l}(z)$ denotes the transfer function of the output samples taken in the $j$-th pulse with respect to the samples of the input signal taken in the $l$-th pulse, and $v$ is an integer that coincides with the number of input samples in a time period $m T_{s}$. Realize that $v$ is equal to $p_{k}$ in the particular case of the SC circuit in Fig. 1(a). Therefore, the general transfer function is given by

$$
H(z)=\frac{1}{L} \cdot \sum_{j=1}^{L} H_{j}(z)
$$

Notice that $L$ coincides with $p_{f}$ in the case of the SC circuit in Fig. 1. By observing eq. (3), it can be seen that the PNIS scheme modifies the transfer function of an SC circuit with respect to the uniform sampling counterpart. In general, PNIS introduces new zeroes and poles. The former do not affect significantly the passband frequency response of the circuit, since they occur at frequency positions larger than the first repetition frequency, and the latter arise as a consequence of a lower effective sampling frequency. For example, if the damped SC integrator in Fig. 1(a) operates with a traditional uniform sampling (i.e., $\phi_{1 \mathrm{f}}=\phi_{1 \mathrm{k}}=\phi_{1}$ and $\phi_{2 \mathrm{f}}=\phi_{2 \mathrm{k}}=\phi_{2}$ ), its transfer function is:

$$
H(z)=\frac{b \cdot z^{-1}}{1-a \cdot z^{-1}}
$$

where the coefficients $a$ and $b$ denote $C /\left(C+C_{2}\right)$ and $C_{1} /\left(C+C_{2}\right)$, respectively. By contrast, if we assume now that the integrator operates with a PNIS, such as $m=4, p_{k}=3$, and $p_{f}=1$, a routine analysis based on the charge conservation law, leads to the following expression of the general transfer function:

$$
H(z)_{P N I S}=\frac{b \cdot z^{-2} \cdot \sum_{i=0}^{2} z^{-l}}{1-a \cdot z^{-4}}
$$

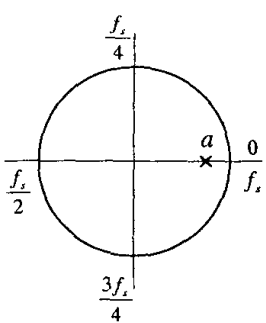

(a)

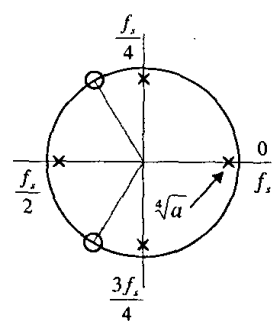

(b)

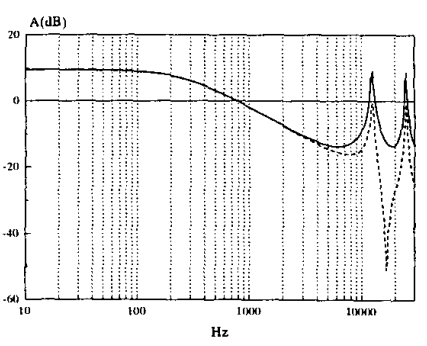

(c)

Fig. 2. Pole-zero pattern representation in the z-plane with uniform sampling (a) and PNIS (b), and the corresponding simulated frequency responses (c) for uniform sampling (solid line) and PNIS (dashed line)

where the unit delay period is still relative to the period of the master clock, $T_{s}=1 / \mathrm{f}_{\mathrm{s}}$. In particular, for the given values of the programming indexes, PNIS generates three additional poles and two zeroes. In order to compare both frequency responses, the damped SC integrator was simulated with a sampling frequency equal to $12.5 \mathrm{kHz}$ for the traditional sampling and with $f_{s}=50$ $\mathrm{kHz}$ for the PNIS sampling. With these operation frequencies, the first repetition frequency occurs at $12.5 \mathrm{kHz}$ in both schemes. Moreover, for uniform sampling the capacitor values were $C_{l}=$ $0.9 \mathrm{pF}, C_{2}=0.3 \mathrm{pF}$, and $C=2 \mathrm{pF}$, whereas for PNIS operation $C_{l}=C_{2}=0.3 \mathrm{pF}$, and $C=2 \mathrm{pF}$. Figures. 2(a) and 2(b) depict the pole-zero location in the $z$-plane for eqs. (4) and (5). Figure 2(c) illustrates the simulated frequency responses of the circuit in Fig. 1 operating with uniform sampling and PNIS.

\section{TIME-MULTIPLEXED SC CIRCUITS WITH PNIS}

Time-sharing or time-multiplexed SC circuits have been shown as an efficient technique for implementing filter banks (parallel time-multiplexing) [2, 3] and high-orders filters (series timemultiplexing) $[4,5]$, in those applications where power and silicon area need to be reduced. The proposed technique based on PNIS can also be used to implement high-performance programmable and fixed-response TM SC circuits. The basic idea behind this approach consists of dividing the available time in time-slots. In every time-slot, the number of pulses in which the different $\mathrm{SC}$ branches are active, can be used to control the parameters of the response for each channel. Needless to say, as in any TM circuit, for each channel, the samples corresponding the output signal of any integrator, must be stored in a respective integrating capacitor until this channel is again active. 


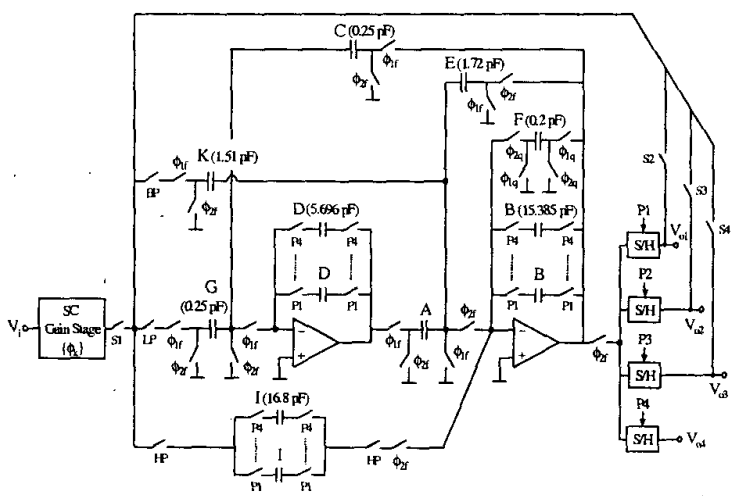

Fig. 3. Circuit schematic of the fabricated TM SC filter programmed with PNIS.

\section{EXPERIMENTAL RESULTS}

To validate the effectiveness of the proposed technique, a universal second-order TM SC filter (4 channels) programmable by PNIS, has been designed. The circuit schematic is shown in Fig. 3. As observed, the filter can provide BP, LP, and HP responses, by enabling appropriate signal paths. The circuit has been fabricated in a $1.2 \mu \mathrm{m}$ CMOS technology with two polysilicon layers. The design was performed to operate with $\pm 1.5 \mathrm{~V}$ supply voltages. The periodical nonuniform programming clock signals $\left(\phi_{\mathrm{f}}, \phi_{\mathrm{k}}, \phi_{\mathrm{q}}\right)$ that control the cutoff or central frequency, $f_{o}$, the maximum gain, $k$, and the $Q$-factor, $Q$, were generated externally by using a commercial FPGA device. In addition, to provide independent programmability of the maximum gain for any type of response, we adopted the solution of including a PNIS programmable offset-compensated SC gain stage [1] in front of the SC filter itself. Every switch $P_{i}$ in Fig. 3 corresponds to one the four channels when the SC circuit operates in TM mode. Switches $P_{i}$ are active sequentially during any time period $m T_{s}$ and, obviously, when the circuit acts as a stand-alone (i.e., single-channel second-order) filter, just one switch is permanently active, while the rest of them are OFF. On the other hand, switches denoted as $\mathrm{S}_{\mathrm{i}}$ only work when the SC circuit operates in series TM mode. In this case, switches $S_{i}$ are controlled by the same clock signals as $\mathrm{P}_{\mathrm{i}}$. Then, the input signal samples, after being processed by the initial SC gain stage, are sequentially computed according to the transfer function realized by each channel. Thus, a transfer function that coincides with the product of the partial transfer functions of the different channels, is implemented. Of course, for any other circuit operation (such as a single-channel stand-alone filter and parallel TM), the switch $S_{1}$ is permanently $O N$, while the rest of the switches $S_{i}$ are OFF.

To provide the SC filter with programmability by PNIS, the sampling frequency for the switched capacitors $\mathrm{A}, \mathrm{C}$, and $\mathrm{E}$ must be used for controlling $f_{o}$, while the sampling frequency of the $\mathrm{SC}$ branch $\mathrm{F}$ will be used for programming the $Q$-factor. The expressions for $k, f_{o}$, and $Q$ are, respectively,

$$
k=m-\left(p_{k}-1\right)
$$

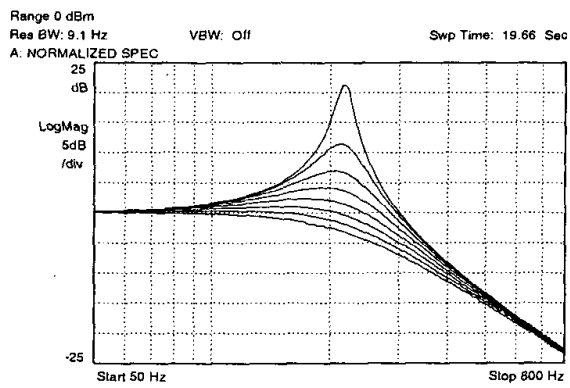

Fig. 4. Experimental $L P$ responses illustrating $Q$-factor programmability $\left(m=8, p_{f}=1,1 \leq p_{q} \leq 8, p_{k}=8\right)$ with $f_{s}=160 \mathrm{kHz}$.

$$
\begin{gathered}
f_{o} \cong \frac{p_{f}}{2 \pi m T_{s}} \cdot \sqrt{\frac{A C}{D(B+E)}} \\
Q \cong \frac{1}{E-\left(p_{q} / p_{f}\right) \cdot F} \cdot \sqrt{\frac{A C(E+B)}{D}}
\end{gathered}
$$

The circuit was designed to operate with a maximum master clock frequency of $1 \mathrm{MHz}$. The capacitor values were chosen in such a way that, when the circuit operates as a stand-alone second-order filter with a master clock frequency $f_{s}=160 \mathrm{kHz}$, it can provide up to eight different responses per magnitude between the limits $0.2 \mathrm{kHz} \leq f_{o} \leq 1.6 \mathrm{kHz}, 0.1 \leq Q \leq 8.9$, and $\mathrm{l} \leq$ $k \leq 8$. That means that the effective sampling frequency for the individual $\mathrm{SC}$ branches varies between $20 \mathrm{kHz}\left(p_{i}=1\right)$ and 160 $\mathrm{kHz}\left(p_{i}=8\right)$. Therefore, in this case, the general programming index $m$ is equal to 8 and the rest of the programming indexes, $p_{k}$ $p_{f}$, and $p_{q}$, can vary from 1 to 8 . Concretely, the capacitor values $\{\mathrm{A}, \mathrm{B}, \mathrm{C}, \mathrm{D}, \mathrm{E}, \mathrm{F}\}$ were determined, from eqs. (6), for satisfying the condition $f_{o}=200 \mathrm{~Hz}, Q=0.707$ with an effective sampling frequency of $20 \mathrm{kHz}$. It should be noted that the value of the $Q$ factor depends on the programmed central or cutoff frequency value. The used design value of $Q$ equal to 0.707 , has been imposed when $p_{f}=p_{q}$. The rest of the capacitors $\{\mathrm{G}, \mathrm{K}, \mathrm{I}\}$ were determined according to the criteria that the filter gain in the frequency passband should be equal to one. After scaling the capacitor values for maximum signal swing and minimum silicon area with a unit capacitor of $0.2 \mathrm{pF}$, the final capacitor values of the SC filter are indicated over the circuit schematic in Fig. 3. Figure 4 shows the experimental $Q$-factor programmability $(1 \leq$ $\left.p_{q} \leq 8\right)$ for the LP response, obtained with $f_{s}=160 \mathrm{kHz}, p_{f}=1$ $\left(f_{o}=0.2 \mathrm{kHz}\right)$ and $p_{k}=8(k=0 \mathrm{~dB})$.

In order to illustrate the versatility of the PNIS programmability, the master clock frequency was set to $1 \mathrm{MHz}$. Figure 5(a) shows the BP responses obtained when varying the programming indexes $p_{f}=p_{q}$ over the entire programmability range $(m=24)$. For Fig. 5(b), $m$ was set to 32 , while the programming index $p_{q}$ swept the available range with $p_{f}=3$. Only the BP responses with a $Q$-factor value larger than 0.707 are included in Fig. 5(b). Notice that in Fig. 5(b) only 22 responses $\left(3 \leq p_{q} \leq 25\right)$ are shown. In this case, for larger $p_{q}$ values, and with the capacitor 


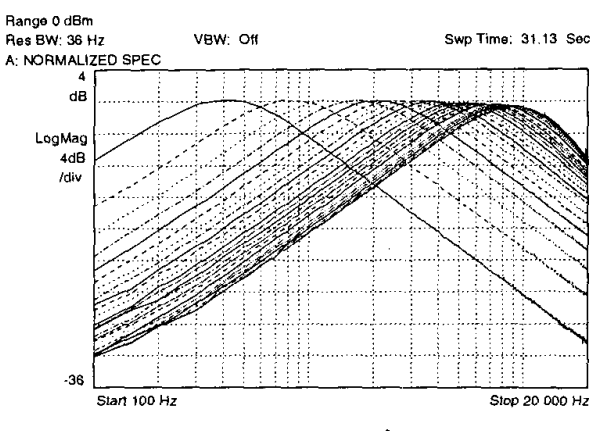

(a)

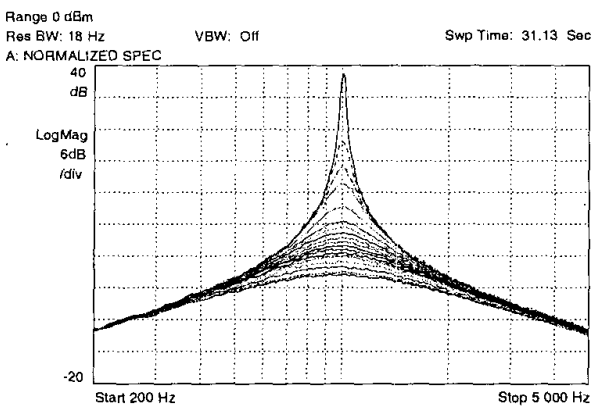

(b)

Fig. 5. Experimental BP responses illustrating central frequency (a) and $Q$-factor (b) programmabilities.

values used, the circuit becomes unstable. According to eq. (6c), the maximum theoretical $p_{q}$ value is 25.8 for this particular programming setting.

Finally, the FPGA circuit was programrned to provide the clock signals necessary for 4-channel series TM operation with $f_{s}=800$ $\mathrm{kHz}$ and $m=8$. Each channel was programmed to provide separately an identical BP response: $f_{o}=1.6 \mathrm{kHz}\left(p_{f}=8\right), Q=1$ $\left(p_{q}=8\right)$, and $k=1\left(p_{k}=8\right)$. Figure 6 depicts the experimental frequency responses. In this plot, the 2-nd order BP response corresponds to the output of the first channel (i.e., $V_{o l}$ in Fig. 3). The 4-th order BP response is obtained at the output of the second channel (i.e., $V_{o 2}$ in Fig. 3) and so on. The measured 8-th order BP response (solid line), which corresponds to the response at the output of the 4-th channel, is compared in Fig. 6 with the ideal simulated response (dotted line). The accuracy of the experimental response is evident.

Unlike the traditional programming techniques for SC circuits, one outstanding advantage of SC circuits programmable by PNIS is that the inherent variable nature of these systems does not necessarily imply a degradation in the optimum dynamic range. To illustrate this aspect, we measured the THD when varying the cutoff frequency of the LP response over a wide programmability range $(m=18)$. A $2-V_{p p}$ sinewave signal was applied to the filter input. To compare the measured THD values in a reliable way,

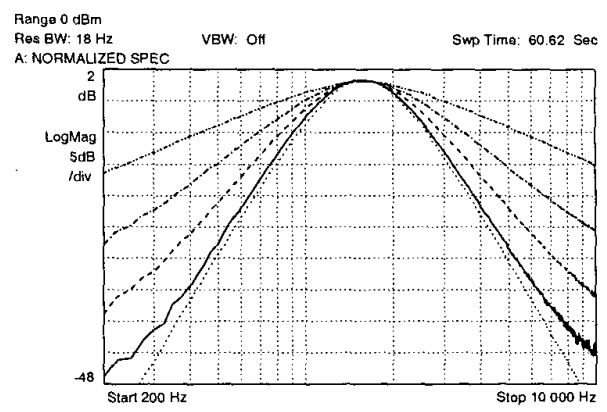

Fig. 6. Experimental BP frequency responses of the four channels obtained with series TM operation. The 8-th order BP response (solid

curve) can be compared with the ideal response (dotted line)

the frequency of the input signal was set equal to the cutoff frequency of the programmed response in each case. With this measurement strategy, all the harmonic frequencies generated at the circuit output were always attenuated by the same factor, regardless of the programmed cutoff frequency value. The measured THD was almost constant ( $\pm 1 \mathrm{~dB}$ of variation) over the entire programmability range, as the voltage swing in any circuit node is not affected by the programmability. Moreover, the power spectral density (PSD) of the output noise was measured in all programmed LP responses, resulting a roughly constant PSD value of $1.3 \mu \mathrm{V} / \sqrt{\mathrm{Hz}}$. Both facts demonstrate that the programmability by PNIS of $f_{o}$ maintains almost constant the circuit dynamic range.

\section{SUMMARY}

The operation of SC circuits with periodical nonuniform individual sampling has been presented. It has been shown that a very wide programmability range in all circuit parameters can be obtained, while the dynamic range degradation is greatly reduced. Also, programmable TM filter banks as well as programmable TM high-order filters, can be easily realized. Experimental results obtained from a test-chip prototype have demonstrated the versatility and potentiality of the technique.

\section{REFERENCES}

[1] R. Gregorian and G. C. Temes, Analog MOS Integrated Circuits for Signal Processing, Wiley, New York, 1986.

[2] J.-C. Bor and C.-Y. Wu, "Analog electronic cochlea design using multiplexing switched-capacitor circuits", IEEE $T$. on Neural Networks, vol. 7, pp. 155-166, January 1996.

[3] J. S. Chang and Y. C. Tong, "A micropower-compatible timemultiplexed SC speech spectrum analyzer design," IEEE J. SolidState Circuits, vol. 28, pp. 40-48, Jan. 1993.

[4] R. Pérez-Aloe, J. F. Duque-Carrillo, E. Sánchez-Sinencio, J. M. Valverde, G. Torelli, and F. Maloberti, "Programmable timemultiplexed switched-capacitor variable equalizer for arbitrary frequency response realizations," IEEE J. Solid-State Circuits, vol 32, pp. 274-279, Feb. 1997.

[5] C.-Y. Wu, J.-C. Bor, and B. S. Jeng, "Realizations of high-order switched-capacitor filters using a multeplexing technique," IEEE T Circ. Syst. II, vol. 41, pp. 778-785, Dec. 1994. 